\title{
Retraction Note to: NPC-16, a novel naphthalimide-polyamine conjugate, induced apoptosis and autophagy in human hepatoma HepG2 cells and Bel-7402 cells
}

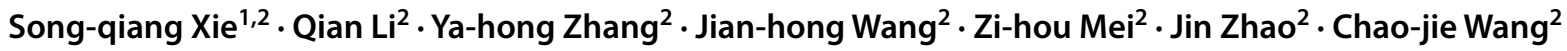

Accepted: 23 January 2021 / Published online: 3 February 2021

(c) Springer Science+Business Media, LLC, part of Springer Nature 2021

\section{Retraction to: Apoptosis (2011) 16:27-34 https://doi.org/10.1007/s10495-010-0537-1}

The Editors-in-Chief have retracted this article [1]. Concerns were raised regarding a number of images, specifically:

Figure $2 \mathrm{~b}$ (Caspase 3 ) appears to partly overlap with Fig. 4g (Cytochrome C (mitochondria)).

Figure 2b (Caspase 9) appears to partly overlap with Fig. 4g (Cytochome C (cytosol)).

Figure 3a (NPC-16 treated HepG2 cells) appear to partly overlap with Fig. 3a (control image of Hela cells) of [2].

Figure 4d (14-3-3) appears to overlap with Fig. 7 (p27Kip1) of [3].

Additionally, there is considerable textual overlap with [2]. The data reported in this article are therefore unreliable.

Authors Chao-jie Wang and Song-qiang Xie disagree with this retraction. The Editor was not able to obtain current email addresses for the other authors.

\section{References}

1. Xie S, Li Q, Zhang Y et al (2011) NPC-16, a novel naphthalimide-polyamine conjugate, induced apoptosis and autophagy in human hepatoma HepG2 cells and Bel-7402 cells. Apoptosis 16:27-34. https://doi.org/10.1007/s10495-010-0537-1

The original article can be found online at https://doi.org/10.1007/ s10495-010-0537-1.

Chao-jie Wang

wcjsxq@yahoo.com

1 Institute of Chemical Biology, Henan University, Kaifeng 475004, China

2 The Key Laboratory of Natural Medicine and Immuno-Engineering, Henan University, Kaifeng 475004, China
2. Wang J, Chen Z, Xie S, Zhao J, Wang C (2010) Synthesis and bioevaluation of aryl-guanidino polyamine conjugates targeting the polyamine transporter. Bioorg Med Chem Lett 20(22):6421-6425. https://doi.org/10.1016/j.bmcl.2010.09.069

3. Tian Z, Xie S, Mei Z, Zhao J, Gao W, Wang C (2009) Conjugation of substituted naphthalimides to polyamines as cytotoxic agents targeting the Akt/mTOR signal pathway. Org Biomol Chem 7(22):4651. https://doi.org/10.1039/b912685f

Publisher's Note Springer Nature remains neutral with regard to jurisdictional claims in published maps and institutional affiliations. 